\title{
Application of a composite measure of product adherence, protocol compliance, and semen exposure to a phase III microbicide HIV prevention trial
}

Terry A. Jacot ${ }^{1 *}$, Meredith R. Clark', Oluwatosin E. Adedipe', Susan Godbout ${ }^{1}$, Tina Cunningham², Susan Ju', Jill L. Schwartz ${ }^{1}$, Sinead Delany-Moretlwe ${ }^{3}$, Andrea R. Thurman ${ }^{1}$ and Gustavo F. Doncel ${ }^{1}$

\begin{abstract}
Background: Strict adherence to antiretroviral-based microbicide use is important for effective HIV prevention. We previously developed a composite measure of product adherence, protocol compliance, and semen exposure for determining vaginal use of tenofovir (TFV) $1 \%$ gel applicators through biomarkers and residual drug analyses. In this study, we tested the ability of the composite measure in vaginally used TFV gel applicators from a Phase III HIV prevention clinical trial.

Methods: Used vaginal gel applicators from the FACTS 001 study were swabbed for detection of vaginal bacterial markers (vaginal insertion), semen DNA markers (semen exposure), and residual TFV gel (product use).

Results: Of 1,098 evaluable TFV and placebo applicators, 80\% had detectable vaginal insertion biomarkers and 52\% had semen biomarkers. Ninety-nine percent of vaginally inserted applicators TFV applicators had detectable residual TFV as measured by liquid chromatography with tandem mass spectroscopy (LC-MS/MS). Residual TFV levels were also successfully detected using Fourier Transform Infrared (FTIR)-based spectroscopy.

Conclusions: Vaginal insertion and semen exposure biomarkers were detectable on used TFV 1\% gel applicators. Residual TFV on these gel applicators was detectable by LC-MS/MS and FTIR-based spectroscopy, which has potential to be a more convenient and quicker method for detecting drug use. With continual improvements, this composite measure of product adherence, protocol compliance, and semen exposure has potential to assess use of not only TFV gel but also other topical microbicides or products.
\end{abstract}

Keywords: Adherence, Biomarker, HIV, Microbicides, PrEP

*Correspondence: jacotta@evms.edu

${ }^{1}$ CONRAD/Department of Obstetrics \& Gynecology, Eastern Virginia

Medical School, VA 23507 Norfolk, USA

Full list of author information is available at the end of the article

(c) The Author(s) 2021. Open Access This article is licensed under a Creative Commons Attribution 4.0 International License, which permits use, sharing, adaptation, distribution and reproduction in any medium or format, as long as you give appropriate credit to the original author(s) and the source, provide a link to the Creative Commons licence, and indicate if changes were made. The images or other third party material in this article are included in the article's Creative Commons licence, unless indicated otherwise in a credit line to the material. If material is not included in the article's Creative Commons licence and your intended use is not permitted by statutory regulation or exceeds the permitted use, you will need to obtain permission directly from the copyright holder. To view a copy of this licence, visit http://creativecommons.org/licenses/by/4.0/. 


\section{Background}

Antiretroviral (ARV) pre-exposure prophylaxis (PrEP) is one option for effective HIV prevention. Topical vaginal delivery (microbicides) is an approach for women who want to avoid the systemic side effects of oral PrEP and who want an on demand regimen. Unlike initial formulations of microbicides that did not show effectiveness, ARV-based microbicides showed promise for PrEP [1]. Tenofovir (TFV) 1\% vaginal gel was tested for effectiveness in preventing HIV acquisition using a single application daily dose or a peri-coital "BAT24" regimen. The BAT24 regimen involved one topical application within $12 \mathrm{~h}$ before sex and one application within $12 \mathrm{~h}$ after sex, and no more than two doses within h. Using the BAT24 regimen, the CAPRISA 004 study in South Africa was the first successful clinical trial that showed a statistically significant reduction of HIV acquisition [2]. In fact, in that same study, the overall effectiveness of $39 \%$ increased to $54 \%$ with high adherence and correct use. However, two additional large clinical trials that followed, VOICE (daily dose regimen) [3] and FACTS 001 (BAT24 regimen) [4], could not confirm these results. In all studies, consistent use at the time of potential exposure was an important factor influencing effectiveness of the gel.
Because self-report is not a reliable method of assessing adherence [5, 6], identifying more objective markers is critical. Microbicide gel use under the BAT24 regimen involves three components: 1) vaginal insertion of the pre-coital gel applicator and expulsion of the gel intravaginally, 2) coital activity (semen exposure), and 3) vaginal insertion of the post-coital gel applicator followed by intravaginal gel expulsion. Methods previously developed to measure vaginal microbicide gel adherence were the ultraviolet light assay and various stain assays utilizing dyes such as trypan blue to detect vaginal fluid on the applicator [7-9]. Since subjectivity of the "readers" could still affect adherence assessments by these methods, we developed a more objective composite measure of product adherence (gel expulsion), protocol compliance (applicator insertion), and semen exposure from a singleuse vaginal gel applicator (Fig. 1), [10]. This composite measure assesses vaginal insertion using vaginal bacteria DNA biomarkers and a protein biomarker, Cytokeratin 4 (CK4). It has been previously shown that immunocytochemical detection of CK4 is specific to vaginal cells that remain on the gel applicator versus epithelial cells of the hand [10]. Our pilot study, CONRAD 125 [11], confirmed that handling the applicator to expel gel outside of the

\section{Composite Measure of Product Adherence, Protocol Compliance, and Semen Exposure for TFV 1\% Gel Applicators}

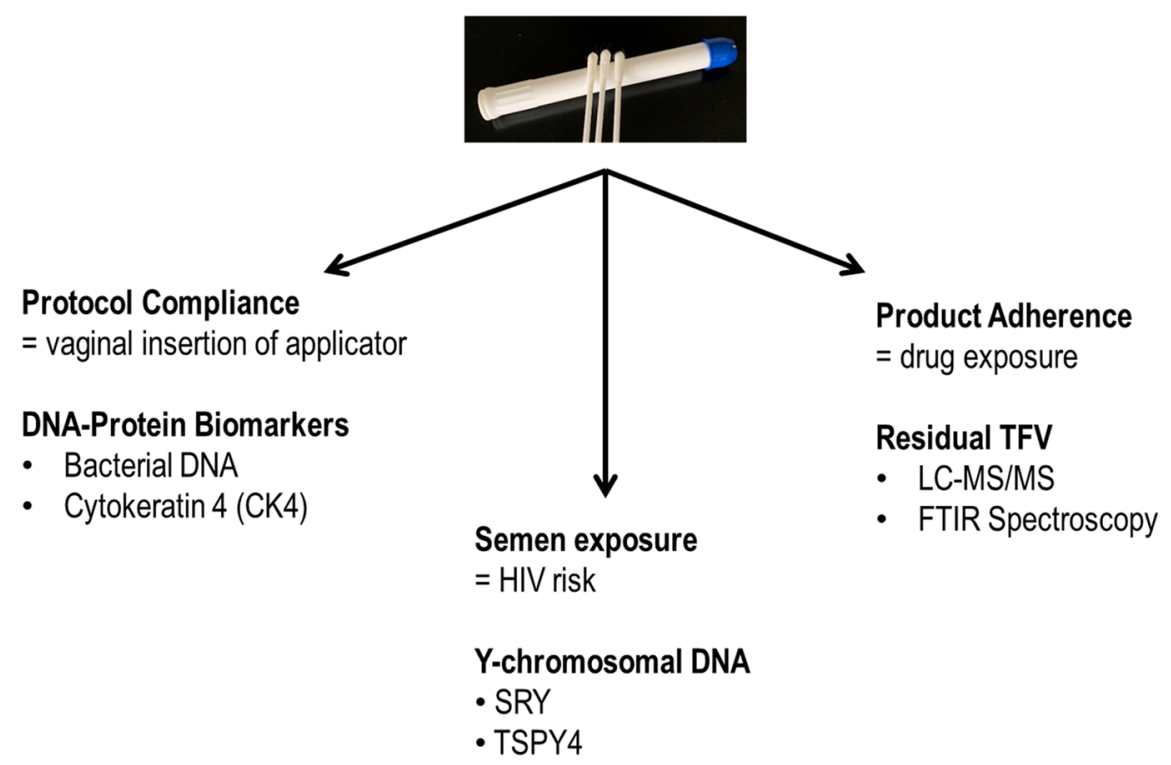

Fig. 1 Individual elements of the composite measure of adherence, protocol compliance, and semen exposure. Returned, used TFV $1 \%$ gel applicators were swabbed with three swab heads to capture biomarkers and residual drug on the barrel of the applicator. The first swab head was used to detect of vaginal bacterial DNA and $y$-chromosomal DNA. The second swab head was used to detect the presence of CK4 in vaginal cells left on the applicator. The third swab head was used for detecting residual TFV by LC-MS/MS and FTIR spectroscopy. More details have been previously published $[10,13]$ 
vagina, i.e., "into the trash", did not have detectable cells or DNA originating from the hand. Semen exposure was assessed through detection of Y-chromosomal DNA biomarkers through a multiplex PCR system $[10,12]$ and gel expulsion through Liquid Chromatography with tandem mass spectroscopy (LC-MS/MS)-based quantitation of residual TFV on the applicator. The vaginal DNA insertion biomarkers were previously validated through a pilot study utilizing directly observed procedures in our clinic [11]. They were more specific and more sensitive than visual inspection of returned applicators and inspection of applicators under ultraviolet light, two methods commonly used for determining applicator use [11].

The FACTS 001 study was a Phase III clinical trial investigating the use of TFV $1 \%$ gel for HIV prevention [4]. Using returned, vaginally used gel applicators from this clinical trial, the primary objective of this substudy was to determine the feasibility of using our composite measure of product adherence, protocol compliance, and semen exposure for a more objective assessment of adherence to these TFV 1\% gel applicators collected in the field during a phase III clinical trial.

In addition, we previously reported the use of Fourier Transform Infrared (FTIR)-based spectroscopy to detect TFV, which would be a faster approach than LC-MS/MS in detecting product for adherence determination in a clinic [13]. Therefore, this exploratory, secondary objective was to test this new approach by measuring TFV on the gel applicators and compare its prediction to LC-MS/MS data.

\section{Methods}

The FACTS 001 study (ClinicalTrials.gov NCT01386294) was a Phase III study conducted at nine clinical sites in South Africa to obtain additional safety and efficacy data for peri-coitally-applied TFV $1 \%$ gel according to the BAT24 regimen [4]. Official clinical trial design and data have been previously published [4]. The study protocol was amended in March 2014 and subsequently approved to collect returned, used vaginal gel applicators for this post-hoc analysis. Participants also signed an additional informed consent to allow the use of their returned applicators. Briefly, participants were given a minimum of 10 individually wrapped placebo or TFV gel applicators to use with sex for one month. Additional applicators were given to the participant depending upon the previous month's self-report of sexual activity. Participants were asked to place used gel applicators into the individual sleeves of provided stripform bags and store in a secure place out of the sun in their homes. Participants then returned their used applicators plus any unused applicators to the clinic at their monthly follow up visit. The applicators were stored in the clinic at room temperature until shipment to the lab at Eastern Virginia Medical School (EVMS). Our laboratory received 5,127 applicators from all nine clinical sites in South Africa, which were collected from participants over a two-month period. The applicator labels did not divulge whether they were placebo or TFV gel, and, therefore, lab personnel analyzed these applicators blinded. Based on available funding and timelines, a representative sample of about 1000 applicators from across all sites was chosen for analysis. To get equal distribution of samples across all nine sites, we began with a random selection of applicators from 10 participants per site. Because participants and sites returned varied numbers of applicators, additional applicators were randomly selected and added to the analysis for a grand total of 1,246 applicators from 141 participants for biomarker analysis. For this analysis set, the number of applicators per site ranged between 104-190 representing 13-21 participants per site (Additional Table 1 ). The only exception was site 07 , which provided applicators from only three participants, 18 applicators total.

Vaginal insertion of the gel applicators were determined by the presence or absence of a panel of vaginal bacterial DNA, human amelogenin DNA, and vaginal cells positive for CK4. Applicators were not considered evaluable if visible mold/bacteria was present or if the patterns of biomarkers were not interpretable. The stability of the biomarkers and resulting ability for their detection was most likely affected, although not uniformly, by the environmental conditions to which the gel applicators were exposed during the time stored between use and shipment to the EVMS laboratory. However, because both DNA and protein markers were used, various patterns of detectable markers were observed and could still be interpreted to determine vaginal insertion (Additional Table 2) Those patterns by which vaginal insertion could not be determined were categorized as not evaluable. Semen exposure was determined by the presence or absence of y-chromosomal (SRY/TSPY4) DNA using the same multiplex PCR amplifying the vaginal insertion DNA markers described above.

Each vaginal applicator was swabbed with three rayon swab heads. The first swab head was used for determining vaginal DNA insertion markers and semen exposure DNA markers. The second swab head was used for assessing the presence of CK4. The third swab head was used for measuring residual TFV by LC-MS/MS. This resulted in swabs from 1,098 evaluable applicators to evaluate vaginal insertion and semen exposure. Of those 1098 applicators, swabs from 901 applicators were chosen for assessment of gel explusion by quantitating residual TFV using LC-MS/ MS. If $100 \%$ of the applicators returned by a single participant had no vaginal insertion biomarkers, those applicators were not included for LC-MS/MS. The lab director was unblinded to treatment groups to assure that the data represented nearly equal numbers of TFV and placebo gel 
applicators. MPI Research Inc. (Mattawan, MI), who performed the laboratory analysis, remained blinded. MPI Research Inc. then returned half of the swabs extracts to the laboratory and a sample of them was analyzed with FTIRspectroscopy analysis.

\section{Vaginal-semen multiplex polymerase chain reaction (PCR) and CK4 immunocytochemistry}

To assess vaginal insertion and semen exposure, one swab was extracted for amplification of vaginal bacterial DNA/Y-chromosomal DNA by multiplex PCR analysis and another swab was extracted for immunocytochemical detection of vaginal epithelial cell protein, CK4. Multiplex PCR and CK4 immunocytochemical detection were performed as previously described [10]. Briefly, the first swab head was placed in DNA extraction buffer. The DNA was isolated and used for subsequent multiplex PCR analysis, which amplifies a panel of vaginal bacterial genes and a human control gene, amelogenin. Because bacteria in the environment could be a contaminating source of DNA, vaginal insertion was confirmed only by co-amplification of one or more vaginal bacteria and the control human gene, amelogenin. The multiplex PCR also includes primers specific for the $y$-chromosomal DNA genes, SRY and TSPY4 [12]. If semen was present on the applicators, residual male DNA would also be amplified. The panel of primers are listed in our previous publication [10].

To detect CK4, the second swab head was placed in $1 \mathrm{ml}$ Cytorich Red solution (Thermo- Scientific, Kalamazoo, MI) for release and fixation of vaginal epithelial cells, which were then spotted onto slides and dried overnight for subsequent colorimetric immunocytochemical detection of CK4. Presence of CK4 positive cells on the applicator was additional confirmation of vaginal insertion.

\section{Measurement of residual TFV on vaginally inserted applicators through LC-MS/MS}

The third arm of our composite measure of adherence was to assess whether gel, which was expelled into the vagina leaving residual gel on the shaft of the applicator, could be measured. After swabbing the used gel applicator, the third swab was stored frozen until shipment to MPI Research, Inc. (Mattawan, MI) where samples were analyzed for TFV concentration. MPI Research developed and qualified the following method specifically for these applicator swab samples. Briefly, contents of the swab were extracted by placing the swab in $1 \mathrm{ml}$ of $\mathrm{dH}_{2} \mathrm{O}$. The extract was split into two aliquots, and to one of them, internal TFV standard was added for LC-MS/MS analysis using the Agilent 1200 Series HPLC in conjunction with Applied Biosystems/MDS SCIEX API 5000 LC-MS/MS System. The calibration curve range was $25.0-25,000 \mathrm{ng} / \mathrm{mL}$ for tenofovir which was equivalent to $25.0-25,000 \mathrm{ng} / \mathrm{swab}$. The other aliquot of the water extract was kept frozen until shipment back to EVMS for FTIR spectroscopy analysis.

\section{FTIR spectroscopy of swabbed TFV and placebo gel applicators extracts \\ Calibration modeling}

Figure 2 summarizes the various steps involved in development and validation of FTIR methods developed to confirm presence or absence of TVF in the swab extracts of the returned gel applicators. The calibration model was developed with a calibration set $(n=60)$ consisting solutions of deionized water containing varying concentrations of TFV 1\% gel and placebo (HEC) gel (1.0, $0.4,0.2$, and $0.1 \mathrm{~g} / \mathrm{mL}$ ) and deionized water to serve as a blank (Fig. 2). A $20 \mu \mathrm{l}$ aliquot of each prepared solution was then placed on a portable Agilent Cary 630 FTIR Spectrometer equipped with a dial path (liquid attachment) accessory (Agilent Technologies, Santa Clara, CA). Spectra of solution containing varying concentrations of active product (40 spectra of TFV gel), placebo product (10 spectra of HEC gel), or no product (10 spectra of deionized water as blank) were captured. The calibration set spectral data were used in developing a partial least square regression (PLSR)-based discriminant algorithm.

\section{Model validation with swab extracts of TFV and placebo gel applicators}

Swab extracts received from MPI Research Inc. were analyzed by placing $20 \mu \mathrm{l}$ aliquot of each swab extract on the FTIR equipment. The operations of the FTIR equipment were controlled by Microlab PC software run from a dedicated computer laptop (Agilent Technologies, Santa Clara, CA). The Microlab PC software was programmed to provide a categorical "Yes" or "No" readouts to confirm presence (Yes) or absence (No) of TVF in the extracts based on discriminant PLSR-based algorithm. FTIR predictions value $>10,000 \mathrm{ng} / \mathrm{ml}$ indicates presence of TFV, and values $\leq 10,000 \mathrm{ng} / \mathrm{ml}$ indicates that the sample is a placebo. Data was collected blinded to the treatment groups.

\section{Statistical analysis}

Descriptive statistics for each outcome are reported. We calculated frequencies and proportions for categorical outcomes. To examine the exploratory objective pilot test on the ability of a previously developed FTIR spectroscopy-based approach to measure TFV from the extracts of the swabbed gel applicators, sensitivity, specificity, and likelihood ratios (both positive and negative) were calculated together with their $95 \%$ confidence intervals. Statistical calculations were performed using R (R Core Team) [14]. 


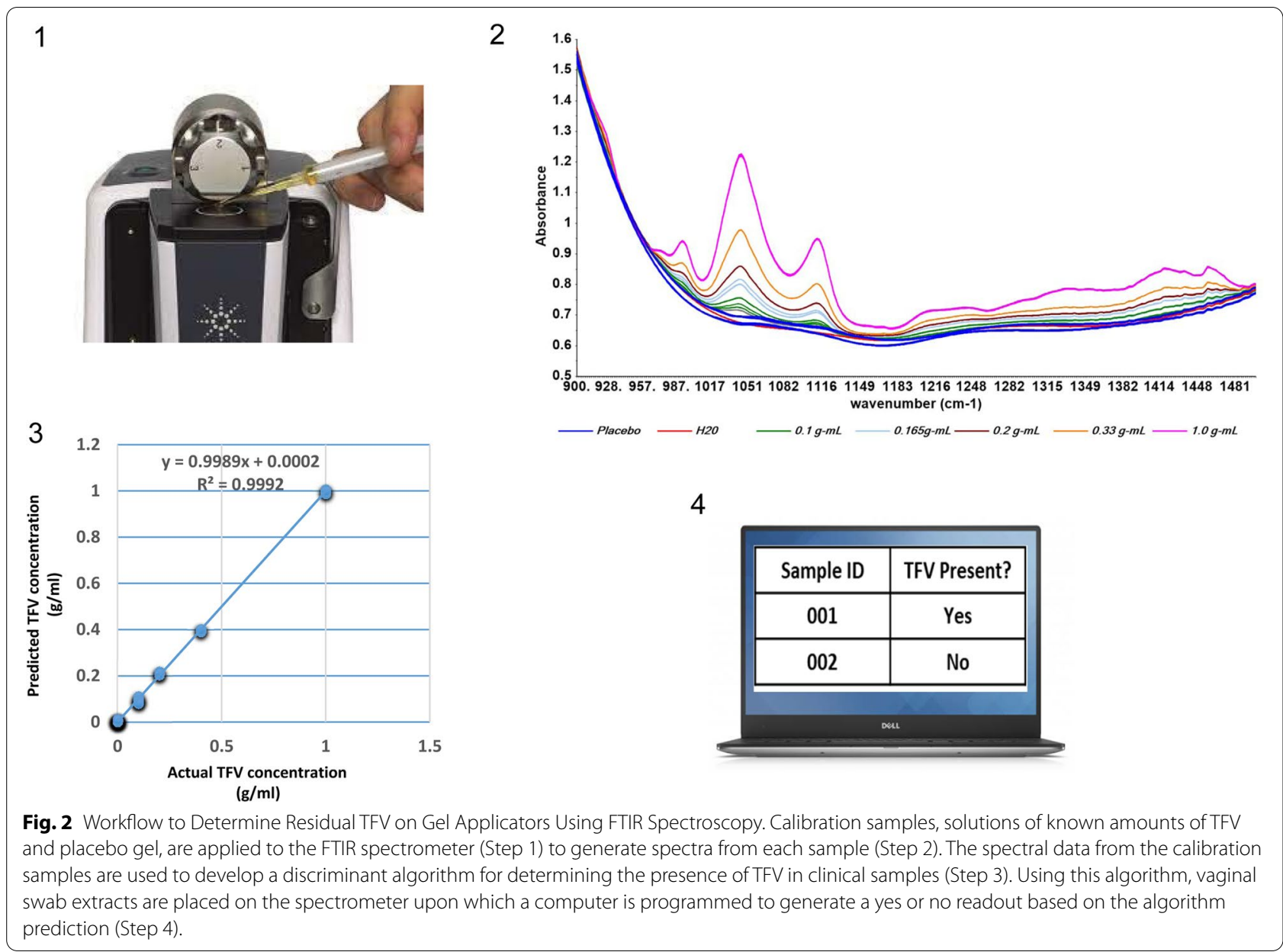

\section{Results}

Out of the randomly selected cohort of 1,246 applicators from 141 participants, 1,098 (88\%) had biomarkers of acceptable quality to evaluate (see criteria in Methods). The median number of returned applicators per woman was eight. Based on vaginal bacterial DNA and CK4 analysis of these evaluable applicators, $80 \%$ (881 applicators) were vaginally inserted while 20\% (217 applicators) were not (Table 1).

The data in Table 1 reflect evaluable applicators in total, which include those applicators from sets returned by participants that were not $100 \%$ evaluable. Therefore, we also did a per participant analysis of applicator use from 101 participants who returned sets of applicators

Table 1 Determination of vaginal insertion of total evaluable applicators

\begin{tabular}{llll}
\hline $\begin{array}{l}\text { Total gel } \\
\text { applicators }\end{array}$ & $\begin{array}{l}\text { Total evaluable } \\
\text { applicators }\end{array}$ & $\begin{array}{l}\text { Total number } \\
\text { of applicators } \\
\text { vaginally } \\
\text { inserted }\end{array}$ & $\begin{array}{l}\text { Total number } \\
\text { of applicators } \\
\text { NOT vaginally } \\
\text { inserted }\end{array}$ \\
\hline $1246(100 \%)$ & $1098(88 \%)$ & $881(80 \%)$ & $217(20 \%)$ \\
\hline
\end{tabular}

that were $100 \%$ evaluable. These participants returned an average of $9 \pm 4$ (mean \pm SD) applicators, which were all evaluable. Of the 101 participants for this analysis, 82 participants returned sets of applicators in which $80-100 \%$ of applicators had detectable insertion biomarker and were deemed vaginally inserted. Further stratification of this group of participants revealed only 12 participants returned sets of applicators for which no biomarkers of vaginal insertion were present and thus considered not vaginally inserted (Table 2 ).

Table 2 Determination of vaginal insertion of applicators from participants returning sets $100 \%$ evaluable $(n=101)$

\begin{tabular}{lll}
\hline $\begin{array}{l}\text { Percent of applicators in the set } \\
\text { deemed vaginally inserted }\end{array}$ & $\begin{array}{l}\text { Number of } \\
\text { participants }(\mathbf{n})\end{array}$ & $\begin{array}{l}\text { Percent } \\
\text { of total } \\
\text { participants }\end{array}$ \\
\hline $0 \%$ & 12 & $11.8 \%$ \\
$1-79 \%$ & 7 & $6.9 \%$ \\
$80-100 \%$ & 82 & $81.2 \%$ \\
Totals & 101 & $100 \%$ \\
\hline
\end{tabular}


Semen DNA biomarkers were detected on $52 \%$ of all evaluable applicators (570/1098). Eight percent (84/1098) of applicators not designated as vaginally inserted had positive detection of semen biomarkers.

The third arm of the composite measure is gel expulsion as measured by residual TFV on the applicator. Of the 1,098 evaluable applicators, extracts of the third swab heads from 901 applicators (556 TFV and 345 placebo) were analyzed to determine if residual TFV gel could be detected by LC-MS/MS (see Methods). The LC-MS/ MS method was highly specific with $97 \%$ (539/556) of the TFV gel applicators having detectable TFV. Only $5 \%(19 / 345)$ placebo applicators were identified as false positives (Table 3). Out of these 901 applicators, 83\% (749) were deemed vaginally inserted by biomarkers. We then assessed the presence of residual TFV on vaginally inserted TFV applicators. TFV was detected on $99 \%$ (452/456) of vaginally inserted TFV applicators (Table 3).

An exploratory objective of this subset analysis was to perform a pilot test on the ability of a previously developed FTIR spectroscopy-based approach to measure TFV from the extracts of the swabbed gel applicators. Using a subset of the original swab extracts described above ( $n=197 ; 122$ TFV, 75 placebo), spectral scans of the extracts were obtained, and yes/no predictions were compared to data generated by LC-MS/MS, the gold standard for this comparison (Table 4). The results from this comparison showed a fairly high sensitivity of 0.83 [95\% CI, 0.75-0.89] and high specificity of 0.97 [95\% CI, 0.91-1.00], with the associated Positive Likelihood Ratio $(\mathrm{LR}+)$ of 31.05 [95\% CI, $7.89-122.14$ ] and Negative Likelihood Ratio (LR-) of 0.18 [95\% CI, 0.12-0.26].

\section{Discussion}

We tested the ability of our previously developed composite measure of product adherence, protocol compliance, and semen exposure to assess adherence to $1 \%$ TFV gel through a post-hoc analysis of gel applicators collected under field conditions from the FACTS 001 study. We report that $80 \%$ of the 1,098 evaluable, returned applicators were vaginally

Table 3 Determination of residual TFV on a subset of returned gel applicators

\begin{tabular}{lll}
\hline All applicators $(\boldsymbol{n}=\mathbf{9 0 1})$ & $\begin{array}{l}\text { Number of } \\
\text { applicators }\end{array}$ & $\begin{array}{l}\text { Number of } \\
\text { applicators with } \\
\text { detectable TFV }\end{array}$ \\
\hline Group & 556 & $539(97 \%)$ \\
\hline P TFV Gel & 345 & $19(5 \%)$ \\
Placebo & 456 & $452(99 \%)$ \\
Vaginally inserted 1\% & \\
TFV gel & & \\
\hline
\end{tabular}

Table 4 Agreement between FTIR-based predictions and LCMS/MS quantitation of residual TFV on gel applicators $(n=197$; 122 TFV, 75 placebo)

\begin{tabular}{llll}
\hline & & \multicolumn{2}{l}{ LC-MS/MS } \\
\cline { 3 - 4 } & & TFV + & TFV- \\
\hline \multirow{2}{*}{ FTIR } & TFV + & 101 & 2 \\
& TFV- & 21 & 73 \\
\hline
\end{tabular}

inserted, while the remainder did not have biomarkers present suggesting non-use. From those evaluable applicators, a subset representing nearly equal numbers of TFV gel and placebo applicators, residual TFV was detected on $97 \%$ of the TFV gel applicators. Combining this with the vaginal insertion biomarker data, we could detect residual TFV in $99 \%$ of the TFV applicators with detectable insertion markers. These TFV gel applicators demonstrate the clinical research potential of these markers to determine objectively that a participant vaginally inserted the applicator and expelled the gel. With a sensitivity of 0.83 and specificity of 0.97 , we also report feasibility of using portable FTIR spectroscopy rather than LC-MS/MS to measure residual TFV on the used applicators.

Unlike our pilot study, CONRAD 125 [11], which analyzed the vaginal insertion markers 7 and 30 days after receipt of applicators in temperature-controlled laboratory conditions, the used gel applicators from the FACTS 001 study were exposed to less controlled environmental storage conditions ("field samples"). For this substudy, participants were asked to store their used applicators at home until they returned them to the clinic at their monthly visits. The applicators were then moved from these uncontrolled conditions to slightly more controlled room temperature conditions at the clinic. Stability of DNA and protein biomarkers are maintained best in cold temperatures. Our initial criteria for vaginal insertion in the pilot validation study was PCR amplification of one or more vaginal bacterial DNA markers and the required amplification of the human control gene, amelogenin, along with positive CK4 immunocytochemical detection. However, testing these "field samples" revealed different patterns of DNA and protein detection because of the different levels of stability inherent in the biomarkers. The ability to detect vaginal bacterial and semen DNA markers was more consistent across samples than CK4 expression in the shed vaginal cells retrieved from the gel applicators. We attributed the inconsistent CK4 observation to variability in the number and quality of the shed cells among participants. Cells with absent CK4 may be due to loss of intracellular contents during the process of cell death and shedding or protein degradation [15]. Although proteins are more specific, they exhibit less stability in harsh environments than DNA. We did not 
consider the possibility of shed cells or DNA from the hand since our previous data showed no detectable, stainable cells or amelogenin DNA amplification from sham applicators, which were applicators simply handled to expel gel into the trash $[10,11]$. Incorporating the human amelogenin gene into the multiplex PCR analysis of vaginal bacteria helped prevent possible false positive determinations of vaginal insertion when the source of bacteria is contamination from the environment. The vaginal microbiome encompasses various bacterial community types ranging from mainly Lactobacilli to more varied anaerobic species [16]. Because of the large diversity of potential bacteria in the vaginal microbiome, one limitation of vaginal insertion biomarkers is the small chance that the multiplex PCR would not amplify any of the chosen bacteria markers in vaginal swab DNA from a minority of participants. In summary, we were able to detect the biomarkers from applicators that were stored for approximately 6-12 months before analysis, and DNA biomarkers were detected more consistently than the protein marker, CK4. While it is best to make a determination with all biomarkers present, it was still possible to make determinations based on different patterns of markers (Additional Table 2). This leads to a general conclusion that the more biomarkers that are available to measure, the more objective ability to assess adherence. For use in smaller clinical trials, e.g., pilot, phase I and phase II studies, optimizing storage and handling conditions through small laboratory controlled studies could clearly improve the assessment of adherence using this composite measure.

A more consistent presence of DNA biomarkers versus protein was also observed in our previous study investigating biomarkers of semen exposure. Y-chromosomal DNA, specifically SRY and TSPY4, was detected on vaginal swabs collected up to $48 \mathrm{~h}$ and 15 days, respectively, from initial time of semen exposure versus prostate-specific antigen [17]. Because multiple copies of the TSPY4 gene are present on the Y-chromosome, it is a very sensitive marker for semen exposure [12]. The current study reports that semen biomarkers were detected in $50 \%$ of the total applicators evaluated, which confirms self-reports of sex acts with no condom use [4]. However, there was a small percentage of applicators deemed not inserted but had detectable TSPY and/or SRY suggesting possible DNA contamination of the applicators during routine manual handling by the participants, particularly when placing the used applicators into the individual sleeves of the provided stripform bags for storage until returning to the clinic. Since the applicators came from an ongoing Phase III clinical trial, we could not acquire an un-inserted applicator stored in those same bags as a control for external contamination from the bags. Our previous validation study used manually handled applicators stored in the original packaging and detected no DNA contamination. In addition, the FACTS 001 study incorporated visual inspection of applicators by clinical personnel into the adherence program, which requires manual handling of the applicators. We acknowledge that these situations added an additional risk for non-specific contamination. While the analysis lab could be another source of DNA contamination, the PCR protocol included a "no DNA" buffer only control to rule out any contamination by the analysis lab. Since applicators were swabbed and DNA extracted from the swabs, a blank swab control was also included in the PCR analysis. While very sensitive, the high contamination risk is a limitation of using TSPY and SRY as markers to determine semen exposure in a study like FACTS 001. A different clinical design plus multiple controls would be necessary to minimize this risk. Another but less likely possibility for unused applicators with only male DNA is large amounts of semen that dilute female DNA/protein to below the threshold of PCR amplification and detection for vaginal insertion markers. This latter possibility, if true, would suggest a false negative (applicator was indeed inserted) and thus a potential underestimate of vaginal insertion. Even with these limitations, the current data confirms that semen markers can be detected on used vaginal gel applicators and would justify further investigation. Smaller, more focused studies involving timed and recorded sex acts are needed to improve their use. Although proteins are less stable, another approach would be future investigations on potential semen-based proteins that could be markers with less contamination risk.

While self-report is not an accurate measure of adherence, more investigative analysis through in-depth interviews (IDIs) and focus group discussions (FGDs) with participants of the FACTS 001 study indicated the challenges of adherence to the BAT24 regimen as it related to the timing and location of sex [18]. Various situational or logistical challenges described by participants resulted in different patterns deviating from the BAT24 regimen: nogel use, pre-coital gel use only, or post-coital gel use only. The presence of semen biomarkers on $50 \%$ of the applicators suggest that nearly half of gel use was post-coital, but it cannot tell us whether those reflect single use or dual gel use as prescribed in the BAT24 regimen. Under the FACTS 001 study design, it was not possible to know which applicator was used pre-coitally or post-coitally. If packaging was designed to provide this information, the biomarkers of adherence could determine the pattern of deviation from the BAT24 regimen and approximately how many sex acts were actually covered by two doses. If we knew whether the unused applicator was a pre- or post-coital dose, that information plus the number of sex acts reported could reveal more accurately whether 1) some sex acts were covered by two doses, 2) every sex act was covered by one dose, or 3) some sex acts were not 
covered by gel at all. To determine semen exposure and correlate it with gel use in future studies, information on which applicator is a pre-coital applicator versus a postcoital applicator would be required.

Although there is no "gold standard" measurement of adherence, a frequent objective marker of adherence is ARV drug levels as quantitated by LC-MS/MS [19-21]. Therefore, swabbing the used gel applicator with an additional swab was done for subsequent extraction and detection of residual TFV. Positive detection of TFV would suggest vaginal expulsion of the gel since previous data of sham applicators (gel expelled but not vaginally inserted) did not have detectable TFV gel [10]. As expected, LC-MS/MS analysis was highly specific detecting residual TFV on TFV gel applicators with only $5 \%$ false positive detection on placebo gel applicators. When combined with the biomarkers of vaginal insertion, TFV was detected in $99 \%$ of the analyzed TFV gel applicators that were deemed vaginally inserted. The qualitative data from the FACTS 001 study reported that some participants admitted to expelling smaller volumes of gel vaginally. This possibility highlights one limitation of our composite measure. We have not tested whether partial amounts of gel vaginally expelled would leave a residue of TFV that is still quantifiable. Overall, the biomarker data suggest that when participants were committed to using the gel applicator, they followed protocol by vaginally inserting the applicator and expelling the gel.

Taking advantage of the LC-MS/MS data, we investigated extending the applicability of our previously published FTIR spectroscopy-based approach that measured TFV gel directly from vaginal swabs [12]. While LC-MS/ MS is a methodology with the high sensitivity and specificity necessary to measure TFV in clinical samples, the results require costly equipment and highly skilled personnel. In addition, results are not provided immediately in a clinic for health care professionals to administer necessary adherence support. Our previous data demonstrated FITR-spectroscopy's potential for non-destructive, immediate analysis of TFV in vaginal swabs. These characteristics are necessary for development of a point-ofcare diagnostic. For this report, we developed a prediction model for determining the presence of TFV in vaginal swab extracts containing residual gel from used applicators. Using those same swab extracts analyzed by LCMS/MS, the qualitative prediction model determined if TFV was present (yes) or not present (no) and compared to LC-MS/MS values, which was set as the gold standard. Statistical methods for estimating sensitivity and specificity as well as likelihood ratios are commonly used for evaluating the performance of a diagnostic test [22, 23]. From this initial data, the LR + estimated that swabs with a positive result (TFV presence) under the LC-MS/MS method is about 31 times more likely to have a positive result under the FTIR method, comparing to those with a negative result under the LC-MS/MS method. Additionally, the LR- showed that swabs with a negative result under the LC-MS/MS method are about 5.6 times to have a negative result under the FTIR method comparing to those with a positive result under the LC-MS/MS method. Accurate likelihood ratios depend upon solid sensitivity and specificity values. Therefore, we recognize that our sensitivity and specificity analysis findings are exploratory only at this stage. A more appropriate sample size is needed to further confirm this conclusion [24]. The pilot data will help in determining the appropriate sample size required for a future study investigating whether FTIR spectroscopy has the promise to be a future approach for a simpler and faster way of measuring ARVs.

\section{Conclusions}

In summary, we demonstrated the feasibility of our composite measure of product adherence, protocol compliance, and semen exposure to objectively assess TFV $1 \%$ vaginal gel use from applicators obtained from a Phase III microbicide HIV prevention trial. With some limitations in stability of biomarkers, we have shown, overall, that these markers have the potential to determine adherence to TFV $1 \%$ gel. With future improvements, combining this type of scientific technology with more in-depth qualitative analysis of adherence, i.e. IDIs and FGDs, will advance the goal of more accurate assessments of correct microbicide use as well as other type of vaginal products using an applicator or device.

\section{Abbreviations}

TFV: Tenofovir; LC-MS/MS: Liquid Chromatography with tandem mass spectroscopy; FTIR: Fourier Transform Infrared; ARV: Antiretroviral; PrEP: Preexposure prophylaxis; BAT24: One topical application within $12 \mathrm{~h}$ before sex and one application within $12 \mathrm{~h}$ after sex, and no more than two doses within 24 h; CK4: Cytokeratin 4; PCR: Polymerase chain reaction; SRY: Sex determining region Y; TSPY4: Testis specific protein Y-linked 4; IDIs: In-depth interviews; FGDs: Focus group discussions.

\section{Supplementary Information}

The online version contains supplementary material available at https://doi. org/10.1186/s41231-021-00100-6.

Additional file 1: Additional Table 1. Number of Applicators Used for Adherence Analysis by Clinical Sites. Stratification of the total number of applicators for analysis by clinical sites

Additional file 2: Additional Table 2. Interpretation of the Adherence Biomarker Patterns to Determine Gel Applicator Use. Different biomarker patterns from vaginal gel applicators

Authors' contributions

Terry A. Jacot - conception/design, acquisition, analysis, and interpretation of data, drafting/revising the manuscript. Meredith R. Clark—conception/design, analysis and interpretation of data, revising the manuscript. Oluwatosin E. Adedipe-design, acquisition, analysis and interpretation of data, revising the 
manuscript. Susan Godbout - acquisition of data. Tina Cunningham —analysis of data, revising the manuscript. Susan Ju-interpretation of data, revising the manuscript. Jill L. Schwartz-interpretation of data, revising the manuscript. Sinead Delany-Moretlwe-interpretation of data, revising the manuscript. Andrea R. Thurman -interpretation of data, revising the manuscript. Gustavo F. Doncel—design, interpretation of data, revising the manuscript. The author(s) read and approved the final manuscript.

\section{Funding}

The FACTS 001 study that provided the gel applicators for the biomarker analysis was funded through the United State Agency for International Development (USAID) under cooperative agreements AID-OAA-A-14-00011 and GPO-A-00-08-00005, the Bill and Melinda Gates Foundation (OPP41266,), and the South African Department of Science and Technology and Department of Health. The applicator biomarker work was funded by USAID under cooperative agreements, AID-OAA-A-14-00011 and AID-OAA-A-14-00005. The contents are the sole responsibility of the authors and do not necessarily reflect the views of their institutions, PEPFAR, USAID or the United States Government. The funders had no role in study design, data collection and analysis, decision to publish, or preparation of the manuscript.

\section{Availability of data and materials}

All data generated or analysed during this study are included in this published article and its supplementary information files.

\section{Declarations}

\section{Ethics approval and consent to participate}

The authors state that they have obtained appropriate institutional review board approval or have followed the principles outlined in the Declaration of Helsinki for all human investigations. Informed consent was obtained from the participants involved in the FACTS 001 study including those participants consenting the use of their returned, used gel applicators for the biomarker analysis.

\section{Consent for publication}

I certify that the work submitted for publication is original and not being considered elsewhere. All co-authors have knowledge of this work and agree to its submission.

\section{Competing interests}

None of the authors have any relevant financial and/or nonfinancial relationships to disclose.

\section{Author details}

${ }^{1}$ CONRAD/Department of Obstetrics \& Gynecology, Eastern Virginia Medical School, VA 23507 Norfolk, USA. ${ }^{2}$ School of Health Professions, Eastern Virginia Medical School, Norfolk, VA, USA. ${ }^{3}$ WITS RHI, University of Witwatersrand, Johannesburg, South Africa.

Received: 29 June 2021 Accepted: 31 August 2021

Published online: 14 September 2021

\section{References}

1. Vyslouzil J, Kubova K, Tkadleckova VN, Vetchy D. Clinical testing of antiretroviral drugs as future prevention against vaginal and rectal transmission of HIV infection - a review of currently available results. Acta Pharm. 2019;69(3):297-319.

2. Abdool Karim Q, Abdool Karim SS, Frohlich JA, Grobler AC, Baxter C, Mansoor LE, et al. Effectiveness and safety of tenofovir gel, an antiretroviral microbicide, for the prevention of HIV infection in women. Science. 2010;329(5996):1168-74.

3. Marrazzo JM, Ramjee G, Richardson BA, Gomez K, Mgodi N, Nair G, et al. Tenofovir-based preexposure prophylaxis for HIV infection among African women. N Engl J Med. 2015;372(6):509-18.

4. Delany-Moretlwe S, Lombard C, Baron D, Bekker LG, Nkala B, Ahmed K, et al. Tenofovir $1 \%$ vaginal gel for prevention of HIV-1 infection in women in South Africa (FACTS-001): a phase 3, randomised, double-blind, placebo-controlled trial. Lancet Infect Dis. 2018;18(11):1241-50.
5. van der Straten A, Montgomery ET, Hartmann M, Minnis A. Methodological lessons from clinical trials and the future of microbicide research. Curr HIV/AIDS Rep. 2013;10(1):89-102.

6. Woodsong C, MacQueen K, Amico KR, Friedland B, Gafos M, Mansoor L, et al. Microbicide clinical trial adherence: insights for introduction. J Int AIDS Soc. 2013;16:18505.

7. van der Straten A, Cheng H, Mensch B, Friedland B, Katzen L, Littlefield S, et al. Evaluation of 3 approaches for assessing adherence to vaginal gel application in clinical trials. Sex Transm Dis. 2013;40(12):950-6.

8. Moench TR, O'Hanlon DE, Cone RA. Evaluation of microbicide gel adherence monitoring methods. Sex Transm Dis. 2012;39(5):335-40.

9. Hemmerling A, Harrison WG, Brown JM, Moscicki B, Oziemkowska M, Bukusi EA, et al. Trypan blue staining to determine vaginal exposure in two types of plastic vaginal applicators containing two different microbicide formulations. Sex Transm Dis. 2012;39(9):710-2.

10. Jacot TA, Nelson A, Thurman A, Kashuba AD, Archer DF, Doncel GF. Development of a composite measure of product adherence, protocol compliance, and semen exposure using DNA and protein biomarkers for topical HIV prevention studies. PLoS One. 2014;9(12):e114368.

11. Thurman AR, Jacot TA, Kimble TD, Mauck C, Nelson A, Schwartz JL, et al. Comparison of visual and ultraviolet light inspection versus DNA/protein biomarkers to assess product adherence with vaginal microbicide applicators. Sex Transm Dis. 2014;41(12):739-46.

12. Jacot TA, Zalenskaya I, Mauck C, Archer DF, Doncel GF. TSPY4 is a novel sperm-specific biomarker of semen exposure in human cervicovaginal fluids; potential use in HIV prevention and contraception studies. Contraception. 2013;88(3):387-95.

13. Adedipe OE, Jacot TA, Thurman AR, Doncel GF, Clark MR. Rapid measures of user's adherence to vaginal drug products using attenuated total reflectance Fourier transform infrared spectroscopy (ATR-FTIR) and multivariate discriminant techniques. PLoS One. 2018;13(5):e0197906.

14. R: A language and environment for statistical computing. Vienna, Austria 2014 [cited 2021]; Available from: http://www.R-project.org.

15. Anderson DJ, Marathe J, Pudney J. The structure of the human vaginal stratum corneum and its role in immune defense. Am J Reprod Immunol. 2014;71(6):618-23.

16. Ravel J, Gajer P, Abdo Z, Schneider GM, Koenig SS, McCulle SL, et al. Vaginal microbiome of reproductive-age women. Proc Natl Acad Sci U S A. 2011;108(Suppl 1):4680-7.

17. Thurman A, Jacot T, Melendez J, Kimble T, Snead M, Jamshidi R, et al. Assessment of the vaginal residence time of biomarkers of semen exposure. Contraception. 2016;94(5):512-20.

18. Scorgie F, Stadler J, Baron D, Ju S, Ikaneng T, Mabude Z, et al. "It was not my aim to sleep there": The impact of timing and location of sex on adherence to coitally-dependent HIV Pre-exposure prophylaxis. AIDS Behav. 2018;22(11):3692-704.

19. Castillo-Mancilla JR, Haberer JE. Adherence measurements in HIV: New advancements in pharmacologic methods and real-time monitoring. Curr HIV/AIDS Rep. 2018;15:49-59.

20. Castillo-Mancilla JR, Zheng JH, Rower JE, Meditz A, Gardner EM, Prehomme J, et al. Tenofovir, emtricitabine, and tenofovir diphosphate in dried blood spots for determining recent and cumulative drug exposure. AIDS Res Hum Retroviruses. 2013;29:384-90.

21. Hendrix CW, Andrade A, Bumpus NN, Kashuba AD, Marzinke MA, Moore $A$, et al. Dose frequency ranging pharmacokinetic study of tenofoviremtricitabine after directly observed dosing in healthy volunteers to establish adherence benchmarks (HPTN 066). AIDS Res Hum Retroviruses. 2016;32(1):32-43.

22. Hess AS, Shardell M, Johnson JK, Thom KA, Strassle P, Netzer G, et al. Methods and recommendations for evaluating and reporting a new diagnostic test. Eur J Clin Microbiol Infect Dis. 2012;31(9):2111-6.

23. Mandrekar JN. Simple statistical measures for diagnostic accuracy assessment. J Thorac Oncol. 2010;5(6):763-4.

24. Flahault A, Cadilhac M, Thomas G. Sample size calculation should be performed for design accuracy in diagnostic test studies. J Clin Epidemiol. 2005;58(8):859-62.

\section{Publisher's Note}

Springer Nature remains neutral with regard to jurisdictional claims in published maps and institutional affiliations. 